\title{
Repetition priming in visual search: Episodic retrieval, not feature priming
}

\author{
LIQIANG HUANG, ALEX O. HOLCOMBE, and HAROLD PASHLER \\ University of California, San Diego, La Jolla, California
}

\begin{abstract}
Previous research has shown that when the targets of successive visual searches have features in common, response times are shorter. However, the nature of the representation underlying this priming and how priming is affected by the task remain uncertain. In four experiments, subjects searched for an odd-sized target and reported its orientation. The color of the items was irrelevant to the task. When target size was repeated from the previous trial, repetition of target color speeded the response. However, when targetsize was different from that in the previous trial, repetition of target color slowed responses, rather than speeding them. Our results suggest that these priming phenomena reflect the same automatic mechanism as the priming of pop-out reported by Maljkovic and Nakayama (1994). However, the crossover interaction between repetition of one feature and another rules out Maljkovic and Nakayama's (1994) theory of independent potentiation of distinct feature representations. Instead, we suggest that the priming pattern results from contact with an episodic memory representation of the previous trial.
\end{abstract}

Visual search is an important component of many activities, from driving in an unfamiliar city to examining $\mathrm{X}$ ray images of baggage at an airport. The task has also proven useful in basic research that has analyzed the underlying mechanisms and limitations of the human visual system (for reviews, see Palmer, Verghese, \& Pavel, 2000; Wolfe, 1998). An impressive aspect of human visual search performance is that, in certain circumstances, search clearly operates in parallel, evaluating many prospective targets at the same time. In these situations, adding more distractors to the scene may have virtually no effect on response time (RT). For example, in the experiments to be reported here, subjects were told to find the odd-sized item and to hit one key if it was tilted in one direction and another if it was tilted the other way. Although on a given trial, the subjects did not know whether the target item would be larger than all the other items in the scene or smaller than all the others, their performance was nevertheless little affected by the total number of items in the scene, suggesting a parallel search.

\section{Priming in Visual Search}

The subject of the experiments described here is the plasticity of visual search mechanisms, as reflected in changes in search performance over successive trials. In

This research was supported by Grant R01 MH45584 from the National Institute of Mental Health to H.P. and by an NEI postdoctoral fellowship awarded to A.O.H. We are grateful to Colin M. Macleod, Anne Hillstrom, and two anonymous reviewers for their useful comments on an earlier version of this manuscript and to Justin A. Junge, Noriko Coburn, and Kristen Sakamoto for assistance with all aspects of the work. Correspondence should be addressed to H. Pashler, Department of Psychology 0109, University of California, San Diego, La Jolla, CA 92093 (e-mail: hpashler@ucsd.edu). experiments by Maljkovic and Nakayama (1994), observers searched for an odd-colored item. On half of the trials, the target was Color 1 and the distractors were Color 2 , whereas on other trials the target was Color 2 and the distractors were Color 1 . Color was the defining feature- the feature that defined which item was the target.

Upon finding the odd-colored item, the observers pressed a key to indicate which of two possible shapes this item had. Interestingly, when the target's color was the same on successive trials, responses were faster, on average, by a few dozen milliseconds. An additional benefit was conferred when the current trial's target color matched the color of the target two, three, or four trials ago. Thus, target color exerted an influence across multiple trials, and the effects of different trials combined approximately additively. When the target on a trial was in the same location as the previous target, responses were speeded even more.

Data in a subsequent article (Maljkovic \& Nakayama, 1996) showed that the effects of repeating the target position and repeating the target color combined approximately additively. To explain all of these data, Maljkovic and Nakayama (1996) theorized that distinct memory elements represent each feature separately and that, on each trial, the "attention-summoning power" of each of the target's features was potentiated. Repetition of the target's shape did not produce any priming, however, and Maljkovic and Nakayama (1996) theorized that only those features relevant to the search were primed (Hillstrom, 2000, added further empirical support for this idea). This observation raises the possibility that repetition priming in visual search may adaptively reflect the task relevance of different features.

The repetition priming effects just described have been shown to be brief in duration, with the features of the tar- 
get affecting RTs significantly only for lags of a few trials (Maljkovic \& Nakayama, 2000). In contrast, a long-term priming effect in visual search has been demonstrated by Chun and Jiang (1998). In their experiments, subjects searched for a rotated letter $\mathrm{T}$ among Ls of random orientation and color. On certain trials, the otherwise random spatial arrangement of distractors and target was repeated, and this yielded a speedup in RTs. Their experiments suggested that an implicit association was learned between the spatial arrangement of distractors and the target location and that several exposures to a particular arrangement was enough to facilitate performance a full week later (Chun \& Jiang, 2003). This result might suggest that distinct mechanisms underlie the long-term priming in difficult visual search shown in Chun and Jiang and the shortterm repetition priming in easy search shown in Maljkovic and Nakayama (1994).

A fundamental issue in visual search is the nature of the representations that the parallel search processes operate on. In some well-known articles, Treisman has suggested that parallel preattentive search operates on an early representation in which such features as color and size are represented separately (Treisman \& Gelade, 1980; Treisman \& Gormican, 1988). Although numerous investigators have countered with evidence that calls into question the existence of early, separate feature representations (e.g., Holcombe \& Cavanagh, 2001; Houck \& Hoffman, 1986), the issue is far from settled. If repetition of different features yields independent additive effects on visual search, as has been suggested by Maljkovic and Nakayama (1996), this would constitute supporting evidence for a stage at which features are represented separately. However, Maljkovic and Nakayama (1996) provided only limited evidence for this. Although they found that the effect of repeating target color and size did not interact with target position, they did not test for the possibility that the effects of repeating nonpositional features might interact.

In the experiments reported here, we investigated the effect of repeating features irrelevant to the search. Finding an effect, we sought to determine whether the benefits of repeating relevant and irrelevant features would combine additively or whether they would interact. In our study, the subjects searched among white and black line segments to find a segment that was odd in its size (it was either larger or smaller than all the other items). The subjects pressed a key to indicate the orientation of this size-discrepant target. The color of the line segments (white or black) was irrelevant to the task.

\section{GENERAL METHOD}

\section{Subjects}

The subjects were undergraduates attending the University of California, San Diego. They participated for credit in a psychology course.

\section{Apparatus}

Stimuli were presented on a $1,024 \times 768$ MAG DX-15T color monitor driven by an Intel Pentium IV 1.8-G machine. The subjects viewed the displays from a distance of about $60 \mathrm{~cm}$ and entered their responses with the keyboard. The size of the display area was about $17 \times 17 \mathrm{~cm}$.

\section{Stimuli}

The search display consisted of 20 items (see Figure 1). In all of the experiments, each item was one of two sizes: 2.2 or $1.1 \mathrm{~cm}$ long. All of the distractors were of one particular length, whereas the target was of another length. The items were randomly arranged within a $17 \times 17 \mathrm{~cm}$ display. Each item was randomly assigned one of two orientations $\left(-45^{\circ}\right.$ or $\left.45^{\circ}\right)$ and one of two colors (white or black, embedded in a gray background). Using the keyboard, the subjects pressed " $\mathrm{j}$ " if the target was $-45^{\circ}$ and " $\mathrm{k}$ " if the target was $45^{\circ}$, using the index and middle fingers of their dominant hands.

\section{Procedure}

The subjects were told to respond as rapidly and as accurately as possible. Each trial began with a small green fixation cross presented in the center of the screen. The subjects were instructed to fixate the cross, which remained present for $400 \mathrm{msec}$, and subsequently to search for the odd-sized item. After a short blank interval (400 msec), the display appeared and remained until the subject responded. A tone (duration of about $200 \mathrm{msec}$ ) sounded to indicate whether the response was correct, and the next trial began $400 \mathrm{msec}$ after the tone offset.

\section{EXPERIMENT 1}

In Experiment 1, the target varied randomly in all three feature dimensions (size, orientation, and color). This method resembled that of previous work (Hillstrom, 2000; Maljkovic \& Nakayama, 1994), but we used one irrelevant dimension (color) that was neither the defining feature (size) nor the reported feature (orientation). This allowed us to ask whether information from such a seemingly task-irrelevant feature would affect the performance.

\section{Method}

Fourteen subjects participated. The color of each item (white or black) was chosen randomly. The background was gray. Whether the

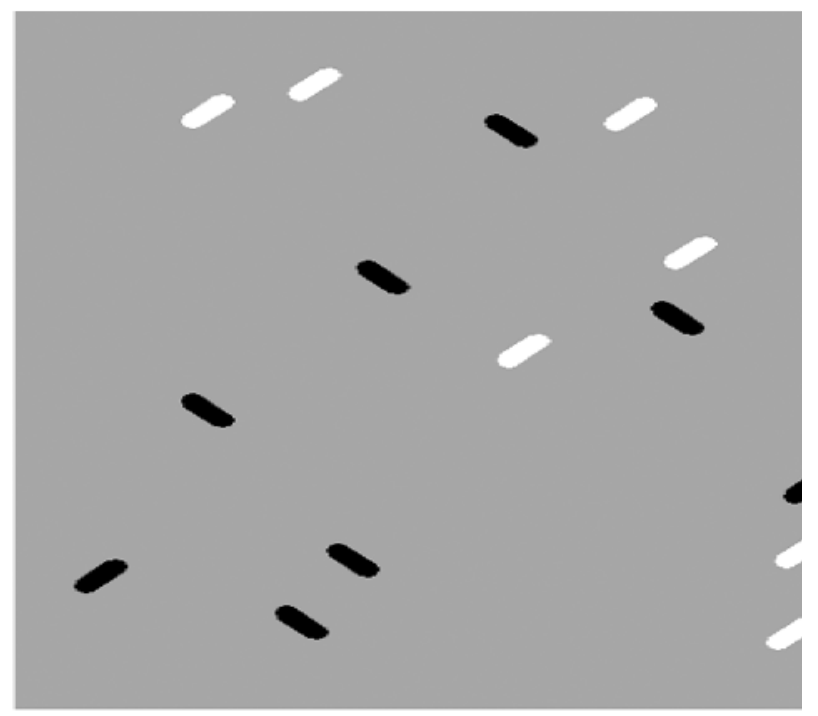

Figure 1. Example of a display used in Experiment 1. The subject attempted to find a line that was longer than the others and responded to the orientation of this line. 
target was white or black, as well as whether it was rightward or leftward tilted and whether it was larger or smaller than all the other items, varied from trial to trial, randomly and independently. All the subjects performed 10 blocks of 100 trials, with the 1st block considered to be practice.

\section{Results and Discussion}

Table 1 shows the error rates in all the experiments, which were quite low. Separate $2 \times 2 \times 2$ analyses of variance (ANOVAs), with repetition of target color, size, and orientation as the main factors, were used to analyze both error rates and RTs. In such an ANOVA, a main effect of size repetition means that error rate or RT is different when two successive targets are the same size from when they are different sizes.

The error rates showed the same trends as the RTs, indicating that none of the effects described here was an artifact of a speed-accuracy tradeoff. With error rates as the dependent variable, the main effect of size repetition was not statistically significant $\left[F(1,13)=3.00, M S_{\mathrm{e}}=\right.$ $0.0003]$. The interaction between repetition of target size and repetition of target color also was not significant $\left[F(1,13)=0.25, M S_{\mathrm{e}}=0.0002\right]$. The interaction between size repetition and orientation repetition was significant $\left[F(1,13)=11.56, M S_{\mathrm{e}}=0.00, p<.005\right]$ and in the same direction as the RT interaction.

An ANOVA on RTs revealed that correct responses were significantly faster when the sizes of successive targets were the same $(888 \mathrm{msec})$ than when they were different $[1,025 \mathrm{msec}$; priming $=137 \mathrm{msec} ; F(1,13)=$ $\left.70.86, M S_{\mathrm{e}}=7,518.50, p<.001\right]$. However, repeating the target orientation [959 msec same vs. $953 \mathrm{msec}$ different, priming $\left.=-6 \mathrm{msec} ; F(1,13)=0.71, M S_{\mathrm{e}}=1,521.19\right]$ or the color [953 msec same vs. $959 \mathrm{msec}$ different, priming $\left.=6 \mathrm{msec} ; F(1,13)=1.40, M S_{\mathrm{e}}=972.07\right] \mathrm{did}$ not yield significant facilitation. Further analysis revealed that the effects of repeating different features was not independent. As is depicted in Figure 2, there was an interaction $\left[F(1,13)=7.59, M S_{\mathrm{e}}=1,882.13, p<.02\right] \mathrm{such}$ that when target size was repeated, repetition of target color $(873 \mathrm{msec})$ resulted in faster responses than when target color was not repeated $(902 \mathrm{msec}$, priming $=$ $29 \mathrm{msec}$ ). However, when target size was different from that in the previous trial, repetition of target color $(1,033 \mathrm{msec})$ actually resulted in slower responses than if the color did not repeat $(1,018 \mathrm{msec}$, priming $=$ $-15 \mathrm{msec}$ ). The implications of this surprising reversal will be discussed shortly, after we consider the effects of repeating target orientation.

In their experiments, Maljkovic and Nakayama (1994) found no effect of repeating the feature that the subjects reported. In our experiments, although repetition of the reported feature, orientation, had no significant main effect, it did interact significantly with repetition of the target size $\left[F(1,13)=11.90, M S_{\mathrm{e}}=1,777.61, p<.005\right]$. As is shown in Figure 2, responses were faster when the target had the same orientation on successive trials, but only if the successive targets were also the same size (877 $\mathrm{msec}$ same vs. $898 \mathrm{msec}$ different, priming $=$ $21 \mathrm{msec}$ ). When the targets on successive trials were of different size, responses were slower if orientation was repeated (1,042 msec same vs. 1,009 msec different, prim-

Table 1

Error Rates (and Standard Deviations) in Experiments 1-4

\begin{tabular}{clcc}
\hline Experiment & \multicolumn{1}{c}{ Condition } & Error Rate $(\%)$ & $S D(\%)$ \\
\hline \multirow{2}{*}{1} & size repeat and color repeat & 3.0 & 2.4 \\
& size repeat and color alternate & 2.7 & 2.5 \\
& size change and color repeat & 3.8 & 2.7 \\
& size change and color change & 3.1 & 3.2 \\
& size repeat and orientation repeat & 2.8 & 2.2 \\
& size repeat and orientation alternate & 3.0 & 2.6 \\
& size change and orientation repeat & 4.5 & 3.5 \\
& size change and orientation change & 2.4 & 1.9 \\
2A & color repeat & 3.4 & 2.2 \\
& color alternate & 3.4 & 2.7 \\
& orientation repeat & 3.5 & 2.4 \\
& orientation alternate & 3.3 & 2.6 \\
& color repeat & 2.9 & 3.0 \\
& color alternate & 2.6 & 3.0 \\
& orientation repeat & 3.0 & 3.2 \\
& orientation alternate & 2.5 & 2.7 \\
3 & color repeat & 3.2 & 3.8 \\
& color alternate & 3.4 & 4.2 \\
& orientation repeat & 4.2 & 4.6 \\
& orientation alternate & 2.4 & 3.0 \\
4 & color repeat (predictable) & 2.5 & 1.8 \\
& color alternate (predictable) & 3.4 & 2.4 \\
& color repeat (random) & 2.4 & 1.8 \\
& color alternate (random) & 2.7 & 1.4 \\
\hline
\end{tabular}



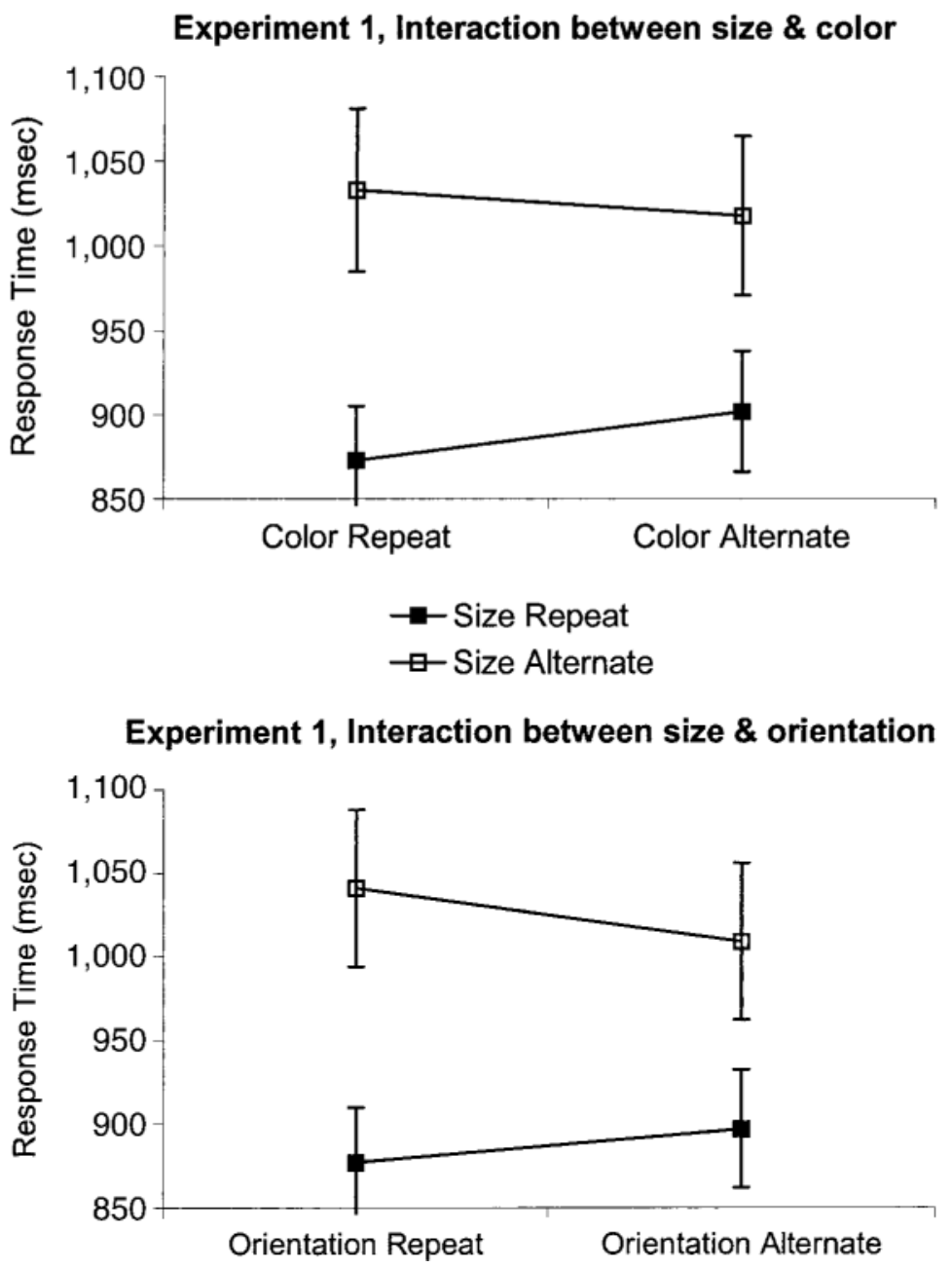

Figure 2. Results of Experiment 1. Although there was little overall priming of the orientation or color dimensions, there were significant interactions of priming of different dimensions.

ing $=-33 \mathrm{msec}$ ). This is the same pattern of interaction as that found for repetition of the target size and target color features. The interaction between repetition of target orientation and repetition of target color was not significant $\left[F(1,13)=1.77, M S_{\mathrm{e}}=439.49\right]$.

The significant interactions shown in this experiment are inconsistent with Maljkovic and Nakayama's (1996) account of repetition priming in visual search. Maljkovic and Nakayama (1996) theorized that presentation of a particular target feature potentiated independent memory elements corresponding to each of the features, leading to independent effects of repeating each feature. But in our experiment, repetition of target color reduced RT only if target size also was repeated. If target size was different from that in the previous trial, RT was actually longer if the target color was the same as that in the previous trial. Simple potentiation of feature representations would imply that each feature increases its power to summon at- tention when repeated and, therefore, that they would work together to determine performance. This would lead to a prediction of an additive effect for different features, with repetition effects for each feature always being positive, contrary to our results.

Another effect present in the data is also inconsistent with Maljkovic and Nakayama's (1996) theory. They suggested that repetition of target features irrelevant to the search would not affect RT, but in the present experiment repetition of target color, despite being irrelevant, did affect RT. In the General Discussion section, we will propose a different, episodic-memory-based theory of priming in visual search.

A critic might argue that rather than disproving Maljkovic and Nakayama's (1996) theory of repetition priming, we have instead discovered a different kind of priming, by tapping into mechanisms different from those operating in the experiments of Maljkovic and Nakayama 
(1996). The experiments described next suggest that the priming documented here, in fact, reflects the same mechanism as that documented by Maljkovic and Nakayama (1996).

\section{EXPERIMENTS 2 AND 3}

Maljkovic and Nakayama (1994) found that repetition priming was unaffected by whether subjects knew the value of the defining feature of the target before each trial began. In other words, when the subjects searched for the odd-colored target, specifying before the trial that the odd color would be red rather than green did not affect the benefit of repeating the target color. Hence, if the priming and interaction found in Experiment 1 is due to the same priming mechanisms as those manifest in the results of Maljkovic and Nakayama (1994), it should occur also when subjects have foreknowledge of the defining feature. To test this, the varying target conditions of Experiment 1 were broken out into separate experiments. In contrast to Experiment 1, in these experiments, the observers always knew whether the target would be smaller or larger than the distractors. In Experiment 2A, the target was always larger than all the distractors; in Experiment 2B, the target was always smaller than all the distractors. In Experiment 3 , the size of the target alternated between larger and smaller on successive trials.

\section{Method}

Eleven subjects participated in Experiment 2A, 9 participated in Experiment 2B, and 14 participated in Experiment 3. In Experiment $2 \mathrm{~A}$, the target was always larger than all the distractors, and the subjects were informed that the target would always be the largest item. In Experiment 2B, the subjects were informed that the target would always be smaller than all the distractors. In Experiment 3, the target alternated on successive trials between being larger and being smaller than the other items. Again, the subjects were informed of this. To avoid any confusion, before each trial began in Experiment 3, the subjects heard an audio recording of the word "big" or "small," which corresponded to the target's size on that trial.

\section{Results and Discussion}

Separate $2 \times 2$ ANOVAs, with repetition of target color and orientation as the main factors, were used to analyze both error rates and RTs. The main effects on RT of repeating different target features are plotted in Figure 3. As in Experiment 1, repeating the irrelevant feature, color, reduced RTs in both Experiment $2 \mathrm{~A}$ [priming $=30 \mathrm{msec}$; $\left.F(1,10)=19.96, M S_{\mathrm{e}}=473.02, p<.001\right]$ and Experiment $2 \mathrm{~B}$ [priming $=56 \mathrm{msec} ; F(1,8)=12.57, M S_{\mathrm{e}}=$ $2,257.16, p<.01]$. However, such repetition did not have a significant effect in Experiment 3, in which the size alternated from trial to trial [priming $=7 \mathrm{msec} ; F(1,13)=$ $\left.1.45, M S_{\mathrm{e}}=1,212.94\right]$. These effects are as expected, as we will discuss. The orientation repetition was not significant in Experiment $2 \mathrm{~A}$ [priming $=16 \mathrm{msec} ; F(1,10)=$ 2.95, $M S_{\mathrm{e}}=1,193.45$ ], Experiment 2B [priming = $18 \mathrm{msec} ; F(1,8)=2.43, M S_{\mathrm{e}}=809.37$ ], or Experiment 3 [priming $=-25 \mathrm{msec} ; F(1,13)=3.31, M S_{\mathrm{e}}=2,678.13$ ].

The interaction between the effects of repeating the color and the orientation was significant in Experiment $2 \mathrm{~A}$ $\left[F(1,10)=5.35, M S_{\mathrm{e}}=654.20, p<.05\right]$ and Experiment $3\left[F(1,13)=5.88, M S_{\mathrm{e}}=783.78, p<.05\right]$, but not in Experiment 2B [ $\left.F(1,8)=0.08, M S_{\mathrm{e}}=650.97\right]$. The direction of the interactions, whether significant or nonsignificant, in each case indicates that repetition of both target features is more advantageous than the sum of the effects of repeating each target feature individually.

The error rates were low, and differences between the conditions were either tiny or in the same direction as the RT differences. In Experiment 2A, when the target color was repeated, errors were marginally fewer $(0.05 \%)$ than when the color was not repeated. In Experiment 2B, the trend was in the opposite direction, but again the effect was miniscule $(0.3 \%)$, so it cannot reflect any important tradeoff between accuracy and RT.

Recall that in Experiments $2 \mathrm{~A}$ and 2B, the size of the target never changed from one trial to the next, whereas in Experiment 3 it always changed. Thus, comparing the ef-

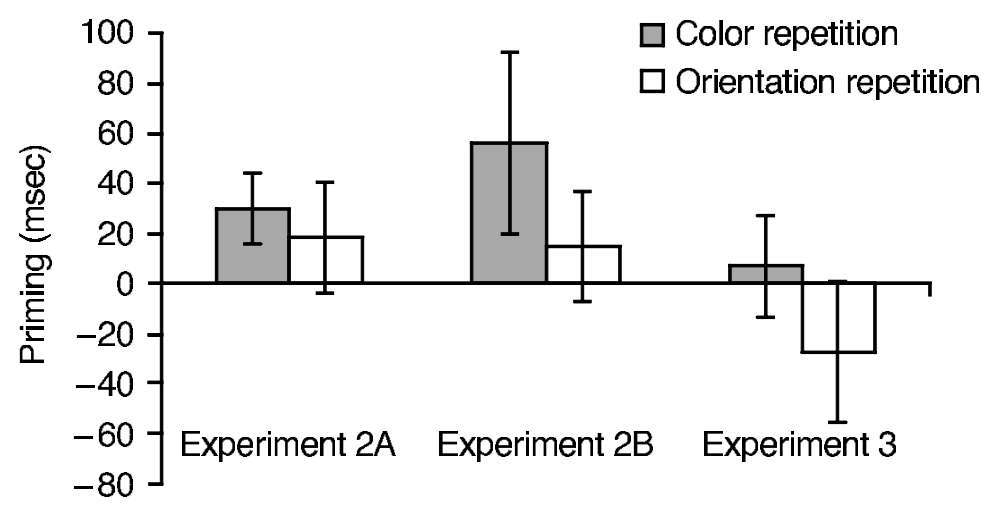

Figure 3. Results of Experiments 2 and 3: color priming and orientation priming. The priming was significantly greater in Experiment 2 than in Experiment 3. 
fect of target color repetition in Experiment 3 with that in Experiment 2 is akin to comparing it with the interaction in Experiment 1: the effect of target color repetition when the target size was repeated versus when it changed. In Experiment 1 , target color repetition was beneficial only when target size also was repeated. In Experiments 2 and 3 , the interaction was not large enough to cause this sort of reversal. However, a between-subjects ANOVA comparing Experiment 2 and Experiment 3 showed that the effect of color repetition when target size changed was less beneficial than when target size was repeated $[F(1,32)=$ 4.68, $\left.M S_{\mathrm{e}}=1,326.26, p<.05\right]$. The benefit of successive targets sharing the same orientation was also smaller in Experiment 3 than in Experiment $2\left[F(1,32)=8.61, M S_{\mathrm{e}}=\right.$ $1,624.20, p<.01]$.

Doing the same across-experiments comparison of error rates, the effect of color repetition was actually in the opposite direction to the RT effect, but the effect was so small [difference $=0.3 \% ; F(1,32)=0.61, M S_{\mathrm{e}}=0.0001$ ] that it is unlikely to reflect any important tradeoff. The orientation repetition effect was consistent in direction with the RT effect and was nearly significant [difference $=1.5 \%$; $\left.F(1,32)=4.00, M S_{\mathrm{e}}=0.0005, p<0.1\right]$.
As in Experiment 1, in these experiments exposure to a target with a particular color primed successive trials. Also as in Experiment 1, the data indicate that the repeating of target color facilitates performance when the target size is also repeated but that the priming is smaller or reversed when the target size changes on successive trials. If this effect of irrelevant target color reflects the same priming mechanism as that seen in the experiments by Maljkovic and Nakayama (1994), it should prime not only the following trial, but also trials stretching farther into the future as well. Indeed, the data from Experiment 2B show that the color of targets before the most recent trial did have an effect. The top panel of Figure 4 shows the effect on RT when a particular preceding trial's target had the same color as the target of the current trial. For example, when the color of the current trial's target was the same as that of the target two trials ago, responses were $42 \mathrm{msec}$ faster $\left[F(1,8)=7.30, M S_{\mathrm{e}}=763.61, p<.05\right]$. This was not true in Experiment $2 \mathrm{~A}$, but in that experiment even the basic priming effect from the immediately preceding trial was much smaller. Hence, it is not surprising that any effect of the preceding trials was undetectable. The bottom panel of Figure 4 provides another look at the
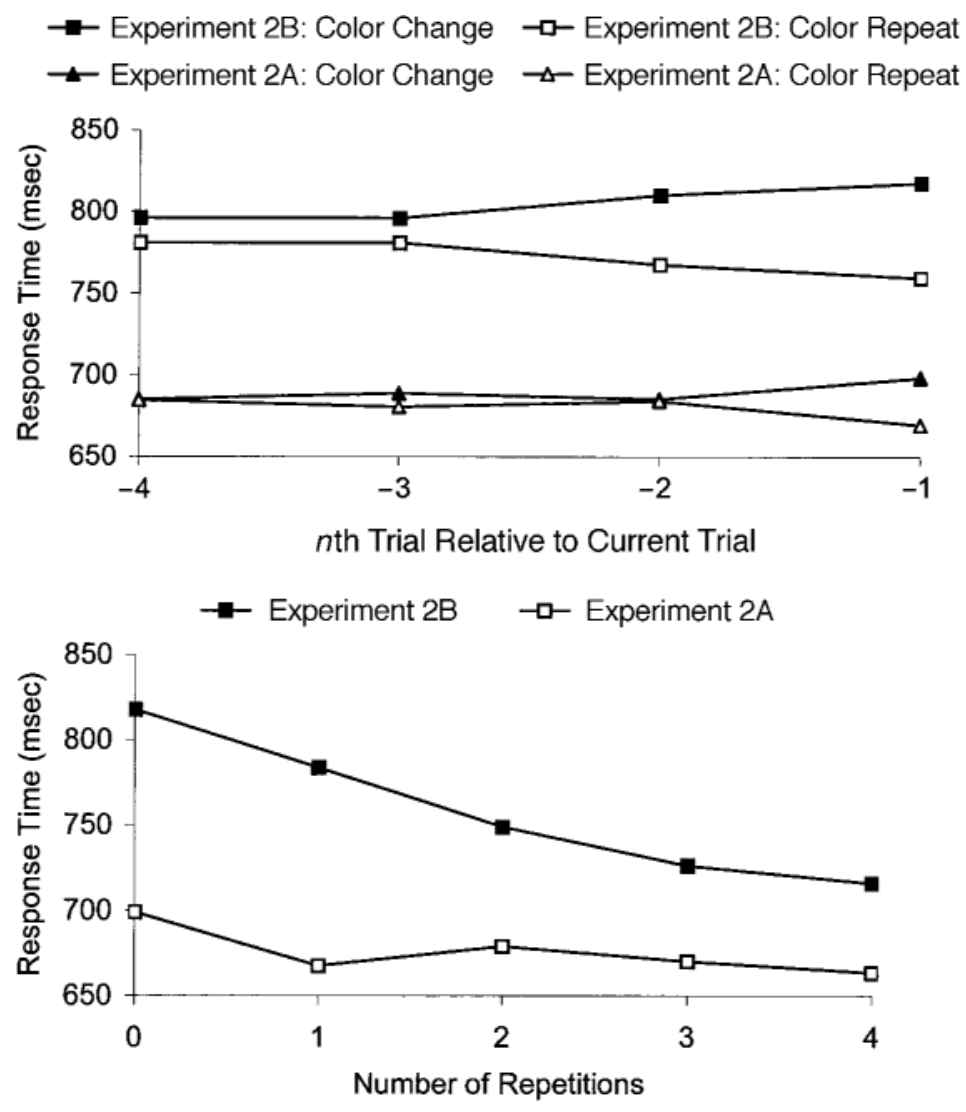

Figure 4. (Top) Effect of the color of the target of previous trials on response times (RTs) in Experiments $2 \mathrm{~A}$ and $2 \mathrm{~B}$. The vertical axis plots the average RT given that the trial $\boldsymbol{n}$ trials ago had a same- or a different-colored target. (Bottom) Cumulative effect of repetitions; the average RT of a trial if it is the last trial in a same-color sequence $n$ trials long. 
influence of previous trials. Rather than showing the influence of particular trials, as in the top panel, the bottom panel shows the accumulating effect for a same-color sequence of different lengths.

\section{EXPERIMENT 4}

The results of Experiments 2 and 3 showed that the priming of target color was little affected by knowledge of the size of the upcoming target. In Experiment 4, we went on to investigate whether repetition priming of target color is affected by foreknowledge of the target color itself. In one condition, the target color was predictable; in the other, the sequence of target colors was random.

\section{Method}

Fourteen subjects searched 20-item displays for the odd-sized target. Items were randomly drawn in white or black and were embedded in the gray background. The target was always bigger than the distractors. All the subjects participated in 10 blocks of 100 trials, with the first 2 blocks excluded from analysis as practice. The blocks alternated between two conditions: predictable versus random. In the predictable block, the target color changed every 2 trials (i.e., white, white, black, black, white, white, etc.). At the beginning of the experiment, the subjects were informed of this and, for the predictable blocks, were told to prepare for the expected target on each trial.

\section{Results and Discussion}

The incidence of errors was analyzed with a $2 \times 2$ ANOVA, with target color repetition and predictability of target color as factors. The main effect of repeating the target color was not significant $\left[F(1,13)=2.58, M S_{\mathrm{e}}=\right.$ 0.0002], and the nonsignificant trend was in the same direction as the RT effects reported next. The interaction between the repetition effect and whether the sequence was predictable also was not significant $[F(1,13)=1.27$, $\left.M S_{\mathrm{e}}=0.0001\right]$.

The same analysis for RT showed that the main effect of repeating the target color was significant [priming $=$ $\left.25 \mathrm{msec} ; F(1,13)=10.86, M S_{\mathrm{e}}=786.12, p<.01\right]$, indicating that responses were faster when the color of the target was the same as that in the previous trial. There was no significant effect of whether the sequence was predictable or random $\left[F(1,13)=0.01, M S_{\mathrm{e}}=1,401.25\right]$. The interaction between predictability and repetition also was not significant $\left[F(1,13)=0.05, M S_{\mathrm{e}}=1,566.78\right]$. So whether the upcoming target color is known or unknown to the subject, the benefit of repeating the target color is similar. This establishes another property in common with the priming investigated by Maljkovic and Nakayama (1994), since they also found that knowledge of the target sequence had no effect on priming.

\section{GENERAL DISCUSSION}

The four experiments described here yielded two theoretically important findings. First, in a task requiring subjects to detect targets on the basis of a size discrepancy, having targets on successive trials share the same color af- fected responses, even though color was irrelevant to the task. This is contrary to Maljkovic and Nakayama's (1994) suggestion that priming occurs only for relevant features. Furthermore, it challenges the idea that priming in this task is always adaptive, since this would imply that only relevant features could prime. Nevertheless, task relevance does affect the magnitude of the priming. The feature that defined the target in our experiments-namely, size- produced much larger priming effects than did taskirrelevant features.

In unpublished work in which a search task different from the present task was used, Maljkovic, Olivola, and Chan (2003) reported substantial effects of task relevance on whether responses are facilitated by feature repetition. Further work must be done to determine under what circumstances task relevance controls priming and to what extent this modulation is useful in natural tasks. One reason for the robust effects of the repetition of irrelevant features in our experiments may be that these irrelevant features belonged to the target objects. Attention to an itemin this case, the target of the search-may automatically trigger processing of all its features, including irrelevant ones (O'Craven, Downing, \& Kanwisher, 1999).

The second important finding of the present experiments was that the effect of repetition of task-relevant target features interacted with the effect of repetition of taskirrelevant target features. For example, in Experiment 1, responses were faster when the target was the same color as the previous trial's target, but only if the target was also the same size as the previous target. When the target was not the same size as the previous trial's target, repetition of target color actually slowed performance. In other words, repetition of target color sometimes resulted in negative priming, rather than positive priming. In some of our experiments, the effect of repeating the target color also interacted with the effect of repeating the target orientation.

These results argue against Maljkovic and Nakayama's (1996) theory that different target features potentiate distinct memory elements, which then combine additively, rather than interacting. Indeed, the interactions discovered here mean that repetition priming in visual search cannot be taken as evidence for Treisman and Gelade's (1980) independent preattentive feature representations. In addition, these results do more than rule out the model in which features are independent. The particular interaction we found indicates that something is going on besides potentiation of separate feature representations. Simple potentiation of representations of features or even representations of pairs of features should never result in detrimental effects from the repetition of any one feature, whatever the values of the other features.

However, another sort of theory, related to one proposed in the literature to explain priming in other tasks, may explain the present results. This theory postulates that repetition effects are caused by the retrieval of an episodic memory representation of the target from the preceding trial. 


\section{Episodic Versus Nonepisodic Explanations of Priming}

Maljkovic and Nakayama (1996) have suggested that detection of a feature increases the ability of that feature to attract attention. For example, after a trial in which the target was red and the distractors were green, red would have increased "attention-summoningpower." As an analogy, Maljkovic and Nakayama (1996) likened the ability of red to attract attention to a capacitor that increases in charge each time a red target is perceived and gradually decreases in charge over time. They further postulated additional capacitor-like memory elements that represent different potential target locations. Processing of the target on each trial would potentiate the capacitor-like element corresponding to the target's location. For features other than location, Maljkovic and Nakayama (1996) proposed that only those that defined the target would be subject to priming. Thus, in search for an odd-colored item, only the color of the target would prime, and in search for an odd-sized item, only the size of the target would prime.

The type of representation described in Maljkovic and Nakayama's (1996) account differs fundamentally from an episodic representation (e.g., Hintzman, 1986; Logan, 1988). Recall that according to Maljkovic and Nakayama's (1996) account, two successive trials with a red target cause two strengthenings of the same capacitor-like memory representation. By contrast, the episodic account postulates the presence of a distinct memory trace for each and every trial, even if the features involved in the current trial are identical to those in the previous trial. This episodic memory trace may include not only a record of certain aspects of the stimulus, but also an association to the strategy used on the preceding trial and the response that was made.

According to this episodic retrieval theory, when the subject begins a new trial, any similarity to occurrences on previous trials can result in retrieval of episodic memories laid down during these prior trials. This retrieval can, in turn, either facilitate or hinder processing on the current trial.

This kind of episodic account has previously been proposed to explain repetition priming and negative priming. In the experiments of Neill (1997), three letters were presented, and observers were told to identify the central letter and ignore the flanking letters. When the central letter on the current trial was identical to the central letter on the previous trial, responses were faster - that is, repetition priming. When the target was a letter that was an ignored flanker on earlier trials, responses were slower than when the target was a novel letter.

Traditional theories of priming have explained these effects with a single preexisting mental representation of the letter identity that becomes more activated in the case of repetition priming or suppressed in the case of negative priming (Morton, 1969; Tipper, 1985)—essentially the same idea as the one that underlies Maljkovic and Nakayama's theory of repetition priming in visual search. But recent results in repetition priming (Hillstrom, 2000) and negative priming have caused problems for the traditional theory. For example, Neill showed that repetition and negative priming effects were contingent on similarity between successive trials. In Neill's experiment, in some trials the target letter was presented at the same time as the irrelevant flankers, and in other trials the flankers appeared $400 \mathrm{msec}$ after the central target letter. The repetition priming effect was larger for successive trials that had the same presentation timing than for successive trials with different presentation timings. Moreover, negative priming did not occur at all when the presentation timing of successive trials was different. The context dependence of the priming in these experiments is difficult to explain simply by activation or suppression of preexisting letter representations.

Neill (1997) proposed an episodic theory according to which the stimuli on a given trial cause the subject to retrieve episodic memories of recent trials that are similar to the present trial. These episodic memories include a record of whether a particular stimulus was responded to or ignored. If the target on the current trial matches the target of the retrieved trial, the episodic record of the retrieved trial facilitates performance. If the target on the current trial does not match the target of the retrieved trial, the memory that the target letter was ignored earlier slows the subject's response. Hence, retrieval of the responded to record can account for the basic repetition priming effect, and the ignored record accounts for the basic negative priming effect. Because the episodic records are hypothesized to also include a memory of the presentation condition, a trial is more likely to result in retrieval of previous trials with the same presentation condition than of those with different presentation conditions. Thus, repetition priming and negative priming are reduced when the last trial has a different presentation condition.

\section{An Episodic Theory of Priming in Visual Search}

The unexpected result of our experiments is that repetition of a target feature can hinder as well as facilitate performance, depending on whether or not the other target features are repeated (i.e., if the size differed, responses were faster if the color or the orientation also differed). Maljkovic and Nakayama's (1996) theory cannot explain this, because their account postulates independent facilitatory priming for each type of repetition. There is no mechanism that could cause repetition of a feature that facilitates performance on some trials but hinders performance on others. But could an episodic theory of priming explain this pattern of effects?

As with other episodic retrieval accounts of priming (Neill, 1997), we propose that on any given trial, episodic memory traces from previous trials are automatically retrieved. This retrieval happens concurrently with the processing of the current stimulus.

What explains the effect of size and the interaction between size and other features? For reasons to be described below, it seems to us that the effect is probably located chiefly at the stage of deciding whether a candidate target 
is, in fact, the target. We assume that when a candidate target has been located, the system does not always proceed directly to choosing the appropriate response. Rather, it seeks to verify that this element is indeed the target. Here, episodic retrieval plays a role. Evidently, the system presumes that when the current potential target matches the episodic memory of a perceived target in terms of target size, the potential target is likely to be the true target. This explains the main effect of target size. Furthermore, we assume that the matching process is not successfully confined to the size dimension, so such properties as color and orientation also affect the duration of the matching process (although not its outcome). When all the feature dimensions cohere in their implications (all favoring the same judgment or all favoring a different judgment), the judgment is easy. On the other hand, inconsistency across dimensions will have a cost. When it arises, the system may need extra time to double check the status of the target. This explains why the repetitions in color and orientation improve performance when size is repeated and impair it when size changes.

\section{Where Does Priming Occur?}

The account just described locates the priming effect at a decision stage that occurs after a candidate target has been found. We assume that the visual search task includes several successive processing stages. The first is searching for the target. The second is the decision just noted. The last is the selection and execution of the response. Could the priming occur during selection and execution of the response? As was mentioned above, size is functionally important in this result. Therefore, the retrieval or comparison cannot occur during response selection or execution, since orientation, not size, is functionally important for those stages. On the other hand, the priming cannot occur only at the perceptual or searching stage, since before the target is found there is no access to its orientation or color, so effects specific to the properties of the target cannot be explained. It should be noted that we cannot completely exclude any role for these stages, but if a single mechanism is proposed to explain all results, the decisional stage appears to be the most likely one.

\section{CONCLUSION}

In the present study, we investigated the effects of the repetition of features in a singleton search task. In addition to replicating the previous finding that repeating the target-defining feature speeds up response, we also found an effect of other nominally irrelevant dimension, and found an interaction between these and the repetition of the target-defining dimension. Unlike in prior accounts of feature priming, we have proposed an episodic memory retrieval account. This account assumes that retrieval of the episode accounts for the main effect of the defining feature (size) and that a comparison between the episode and the present trial explains the effect of the other featural dimensions (color and orientation), as well as the interactions.

\section{REFERENCES}

Chun, M. M., \& JiAng, Y. (1998). Contextual cueing: Implicit learning and memory of visual context guides spatial attention. Cognitive Psychology, 36, 28-71.

CHUN, M. M., \& JIANG, Y. (2003). Implicit, long-term spatial contextual memory. Journal of Experimental Psychology: Learning, Memory, \& Cognition, 29, 224-234.

Hillstrom, A. P. (2000). Repetition effects in visual search. Perception \& Psychophysics, 62, 800-817.

HinTZMAN, D. L. (1986). Schema abstraction in a multiple trace memory model. Psychological Review, 93, 411-428.

Holcombe, A. O., \& CAVAnagh, P. (2001). Early binding of feature pairs for visual perception. Nature Neuroscience, 4, 127-128.

HoucK, M. R., \& HofFMAN, J. E. (1986). Conjunction of color and form without attention: Evidence from an orientation-contingent color aftereffect. Journal of Experimental Psychology: Human Perception \& Performance, 12, 186-199.

LogAn, G. D. (1988). Toward an instance theory of automatization. Psychological Review, 95, 492-527.

MalJKovic, V., \& NAKAYAMA, K. (1994). Priming of pop-out: I. Role of features. Memory \& Cognition, 22, 657-672.

Maljkovic, V., \& NAKAYAma, K. (1996). Priming of pop-out: II. The role of position. Perception \& Psychophysics, 58, 977-991.

Maljkovic, V., \& Nakayama, K. (2000). Priming of pop-out: III. A short-term implicit memory system beneficial for rapid target selection. Visual Cognition, 7, 571-595.

Maljkovic, V., Olivola, C., \& CHAN, D. (2003). Task relevance, not stimulus salience, gates access to implicit working memory. Manuscript submitted for publication.

MORTON, J. (1969). Interaction of information in word recognition. Psychological Review, 76, 165-178.

NEILL, W. T. (1997). Episodic retrieval in negative priming and repetition priming. Journal of Experimental Psychology: Learning, Memory, \& Cognition, 23, 1291-1305.

O'Craven, K. M., Downing, P. E., \& Kanwisher, N. (1999). fMRI evidence for objects as the units of attentional selection. Nature, $\mathbf{4 0 1}$, 584-587.

Palmer, J., Verghese, P., \& Pavel, M. (2000). The psychophysics of visual search. Vision Research, 40, 1227-1268.

TIPPER, S. P. (1985). The negative priming effect: Inhibitory priming by ignored objects. Quarterly Journal of Experimental Psychology, 37, 571-590.

TRE ISMAN, A., \& GELADE, G. (1980). A feature-integration theory of attention. Cognitive Psychology, 12, 97-136.

Treisman, A., \& GormicAn, S. (1988). Feature analysis in early vision: Evidence from search asymmetries. Psychological Review, 95, 15-48. Wolfe J. (1998). Visual search. In H. Pashler (Ed.), Attention (pp 1374). London: Psychology Press.

(Manuscript received October 31, 2002; revision accepted for publication August 7, 2003.) 